\title{
Differential Diagnosis and Management on Seborrheic Keratosis in Elderly Patients
}

\author{
Elvira Moscarella' \\ Gabriella Brancaccio' \\ Giulia Briatico' \\ Andrea Ronchi ${ }^{2}$ \\ Simonetta Piana ${ }^{3}$ \\ Giuseppe Argenziano' \\ 'Dermatology Unit, University of \\ Campania, Naples, Italy; ${ }^{2}$ Pathology Unit, \\ University of Campania, Naples, Italy; \\ ${ }^{3}$ Pathology Unit, Azienda USL-IRCCS di \\ Reggio Emilia, Reggio Emilia, Italy
}

\begin{abstract}
Seborrheic keratoses are exceedingly common in the elderly and usually are easy to diagnose and do not require treatment. However, given their great variety of clinical presentation, they may give rise to false-positive cases, meaning that they may at times mimic melanoma, squamous cell carcinoma and basal cell carcinoma. On the other hand, melanoma may mimic seborrheic keratosis, leading to incorrect patient management. With this review, we would like to summarize the current knowledge about epidemiology, clinical, dermoscopic and reflectance confocal microscopy imaging of this common entity, and we also summarize the currently available treatment options.
\end{abstract}

Keywords: seborrheic keratosis, melanoma, dermoscopy, reflectance confocal microscopy, elderly, basal cell carcinoma

\section{Introduction}

Seborrheic keratosis (SK) is a benign intraepidermal neoplasm arising from epidermal keratinocytes. Also known as senile warts, seborrheic keratoses are very common in aging skin, developing typically on the trunk, head and neck, and in skin creases. ${ }^{1}$

Ultraviolet radiation exposure is considered the most important etiologic factor; other possible causes under investigation include genetic and metabolic factors. . $^{2,3}$

Several histologic subtypes have been described that give rise to a variety of clinical presentations. ${ }^{1}$ Usually, SK presents clinically as solitary or multiple pink to brown flat plaques with a filiform surface (Figure 1). The diagnosis is readily made in the great majority of cases, they are extremely common in the elderly, however, given their high variability in clinical and dermoscopic presentation they may be at times misdiagnosed, mimicking melanoma, squamous cell carcinoma and basal cell carcinoma. ${ }^{4,5}$ On the other hand, melanoma may mimic SK clinically, presenting with a verrucous surface. This is a more worrisome scenario, when a thick nodular melanoma is misdiagnosed and eventually treated as SK. ${ }^{6-8}$ As a general rule, all lesions should be examined by dermoscopy, irrespective of their clinical appearance. This simple rule may avoid missing the aggressive type of nodular melanoma. Reflectance confocal microscopy (RCM) is a third level non-invasive diagnostic tool that may help lesion assessment in challenging cases. ${ }^{9}$
Dermatology Unit, University of Campania, Nuovo Policlinico (edificio 9C), Via Pansini 5, Naples, 80I3I, Italy

Tel +39335 I727229

Email elvira.moscarella@gmail.com 

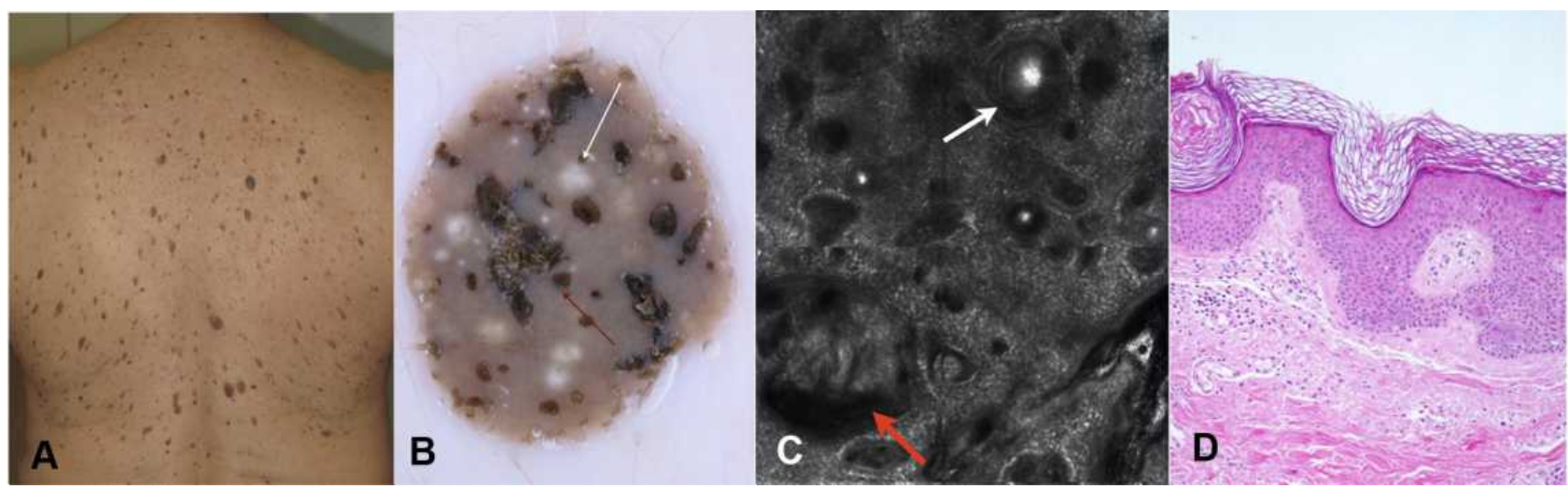

Figure I Clinical, dermoscopy, RCM and histology of an acanthotic SK. (A) Clinical overview of the back of one 70-year-old man with multiple SKs. (B) Dermoscopy of one lesion, multiple milia-like cysts (white arrows) and comedo-like openings (red arrows) are visible. (C) RCM imaging at the level of the supra basal layer, enlarged inter papillary spaces are visible, with horn pseudocysts (white arrows) corresponding to milia-like cysts. The red arrow points a more superficial epidermal invagination filled with keratin, corresponding to a comedo-like opening in histology. (D) Histology showing acanthosis of epidermis and epidermal invaginations filled with keratin (hematoxylin and eosin stain, original magnification 100x).

The management of SK is usually conservative, patients may require lesion removal for either cosmetical or functional reasons. Many therapeutic options are currently available, including cryotherapy, minor surgery and laser removal.

The aim of this review is to encompass all the diagnostic clues that may help the clinician recognize and manage SK, especially through dermoscopy and reflectance confocal microscopy in elderly patients.

\section{Epidemiology}

Accurate and updated information about SK epidemiology are missing; however, it is well known that the prevalence of SK increases with increasing age. A higher prevalence among people with skin of color has also been reported. In a study in US in 1963, Tindall and Smith examined a population of individuals older than 64 years and found that $88 \%$ of the people had at least one seborrheic keratosis. ${ }^{10}$ No sex difference has ever been reported for SKs.

\section{Pathophysiology}

SKs seem to result from a clonal expansion of a mutated epidermal keratinocyte. The most frequently mutated genes in seborrheic keratoses are FGFR 3 (fibroblast growth factor receptor 3) (found in $71 \%$ or sporadic seborrheic keratosis) and the p110 catalytic subunit of phosphatidylinositol 3 kinase (PI3K) (found in $50 \%$ of sporadic seborrheic keratoses). ${ }^{11}$ SKs have a higher proliferative rate than normal keratinocytes, apoptosis is suppressed in SKs compared with healthy skin. Proliferative keratinocytes trigger the activation of neighboring melanocytes by secreting melanocyte-stimulating cytokines in pigmented SKs.

\section{Histopathology}

From a histologic point of view, an SK is composed of a proliferation of basaloid keratinocytes with a papillomatous architecture on a flat base, and in its classical form it shows broad adjoining columns of hyperplastic epidermis (acanthotic type). However, several histological variants are recognized with difference in the extent of hyperkeratosis (keratotic or papillomatous type), hyperparakeratosis and squamous eddies (irritated type) or presence of pigmented keratinocytes and melanophages (pigmented type). When the acanthosis is mild it could be difficult to distinguish macular or reticulated type-SK from solar lentigo. Lastly, clonal type SK is characterized by intraepidermal nests of pale or pigmented basaloid keratinocytes; in its pigmented variant clonal-SK is the most challenging form of SK, being difficult to differentiate from both melanoma and pigmented basal cell carcinoma. ${ }^{1}$

\section{Dermoscopic Features of SK}

Dermoscopy of SK has been extensively described in literature. $^{4,5,12}$ The three main features of SK are:

1. milia-like cysts: roundish white-yellow clods of variable size; they correspond to intraepidermal horn cysts (Figure 1B). 


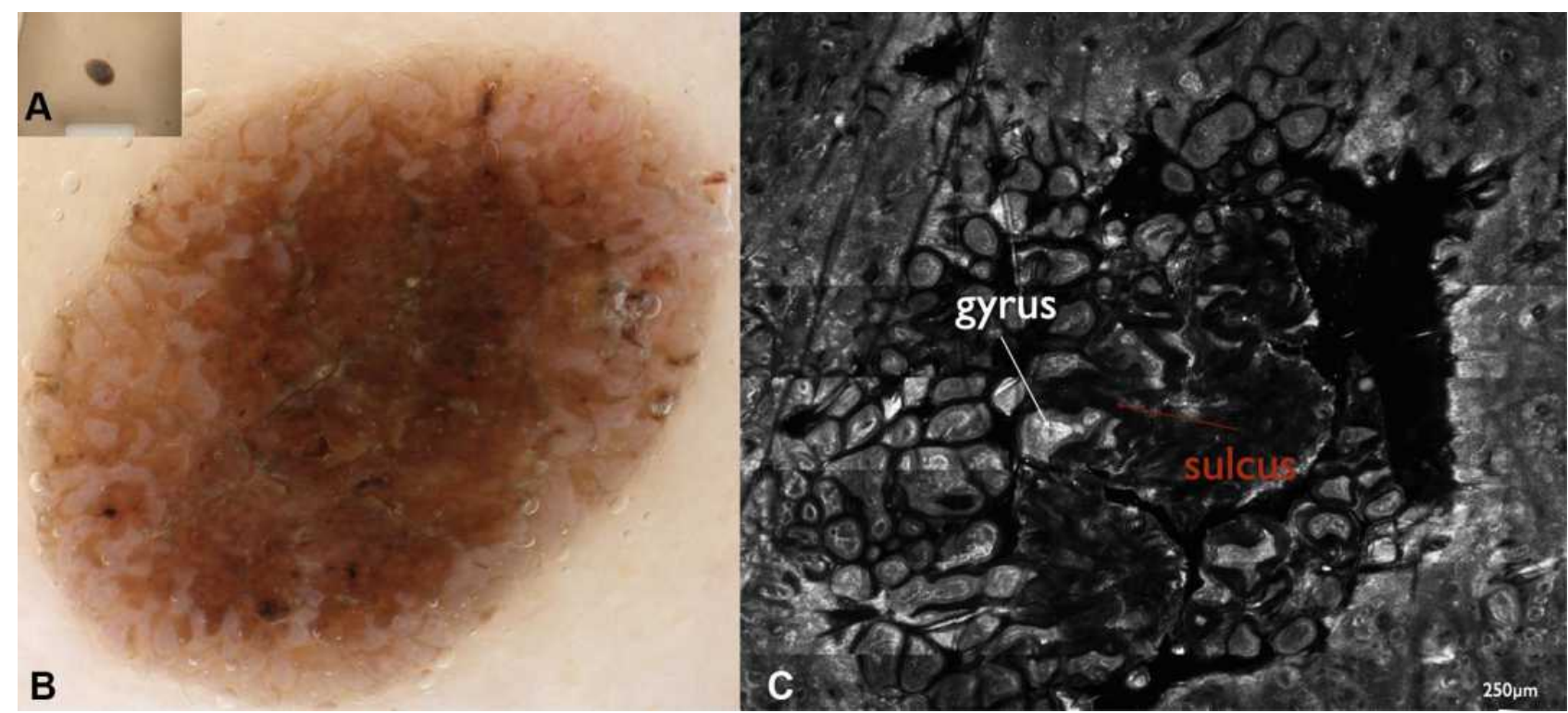

Figure 2 Hyperkeratotic SK. (A) Clinical close up of a brownish pigmented papule with a crusty surface. (B) In dermoscopy a brain-like appearance is visible. (C) RCM mosaic $(5 \times 4 \mathrm{~mm})$ showing multiple epidermal invaginations giving rise to sulci and gyri corresponding to the dermoscopic cerebriform surface.

2. comedo-like openings: well-circumscribed oval or rounded black to brown clods that correspond to keratin aggregates located in the dilated follicular openings (Figure 1B).

3. fissures and ridges: thick, curved, occasionally branched lines whose colors vary from hypopigmented to brown, black and blue. As they give to SK a global appearance that resembles the surface of the brain, they are also named sulci and gyri (Figure 2). The exophytic papillary structures (gyri) are tightly juxtaposed domeshaped structures, generally separated by blackish

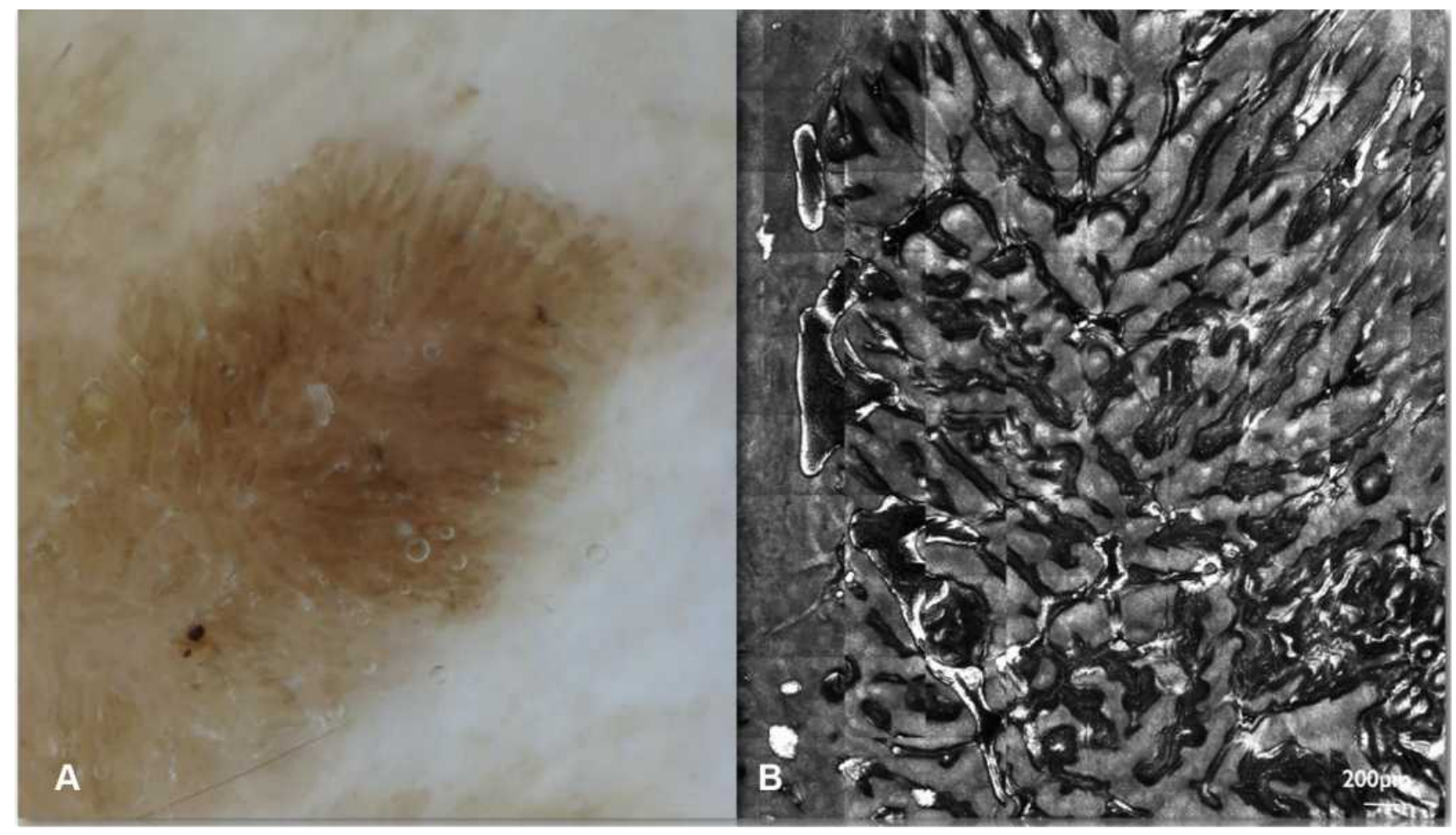

Figure 3 Hyperkeratotic SK. (A) Dermoscopy showing multiple fat fingers, a variation on the theme of cerebriform surface. (B) Corresponding RCM mosaic $(5 \times 3$ mm) showing the lesion surface with multiple sulci and gyri. 
comedo-like openings (irregular furrows or crypts). In fact, comedo-like openings are nothing more than keratin plugs present in the various indentations. Fat fingers are a variation on the theme of cerebriform appearance, they are thick digitate linear, curvilinear, branched, or oval/circular dermoscopic structures typically seen in seborrheic keratoses where they represent the gyri of their cerebriform surfaces. ${ }^{13}$ The "fat finger" gyri are accentuated by pigmented keratin filling the sulci (Figure 3).

These features are often accompanied by sharply demarcated borders (Figure 4) and light brown tiny ridges running in parallel and producing a pattern that is reminiscent of fingerprints (fingerprints structures). Also, the vascular pattern typical of SK is made up by hairpin vessels, described as linear looped vessels forming a half-looped or hairpin-like structure. The vessels are often surrounded by a white halo, this is a typical finding in such keratinocytic neoplasms as SK.

\section{Dermoscopic Mimics of SK}

Milia-like cysts and comedo-like openings are not exclusively of SK. These findings are often present in benign lesions as verrucous dermal nevi, but are described even in melanoma, especially when folliculotropism is present.

There are many reports of verrucous or nodular melanomas clinically misdiagnosed as SKs. ${ }^{14-23}$

In an analysis of 9204 lesions clinically diagnosed as SKs, melanoma was histologically identified in 61 cases. $^{22}$ Carrera et $\mathrm{al}^{23}$ analyzed the dermoscopic appearance of melanomas that clinically simulate SK reporting scaly and hyperkeratotic surface, yellowish keratin, comedo-like openings, and milia-like cysts as the most frequent SKfeatures showed by clinically challenging melanomas. In these cases, the presence of the blue-black sign, pigment network, pseudopods or streaks, and/or blue-white veil, allows the correct diagnosis of most of the difficult melanoma cases. $^{23}$

Basal cell carcinoma (BCC) and SK may coexist as collision tumors. ${ }^{24,25}$ However, BCC may clinically and dermoscopically resemble a SK and vice versa. ${ }^{26,27}$ A retrospective study on histologically confirmed BCCs of the lower limbs showed that they frequently simulate other benign or malignant lesions. Seborrheic keratosislike BCC exhibited hairpin vessels, white-pink areas as well as multiple blue-grey dots and granularity that may be present in regressive SK (lichenoid keratosis, see later). ${ }^{28}$ SK mimicking basal cell carcinoma may present ulcer,

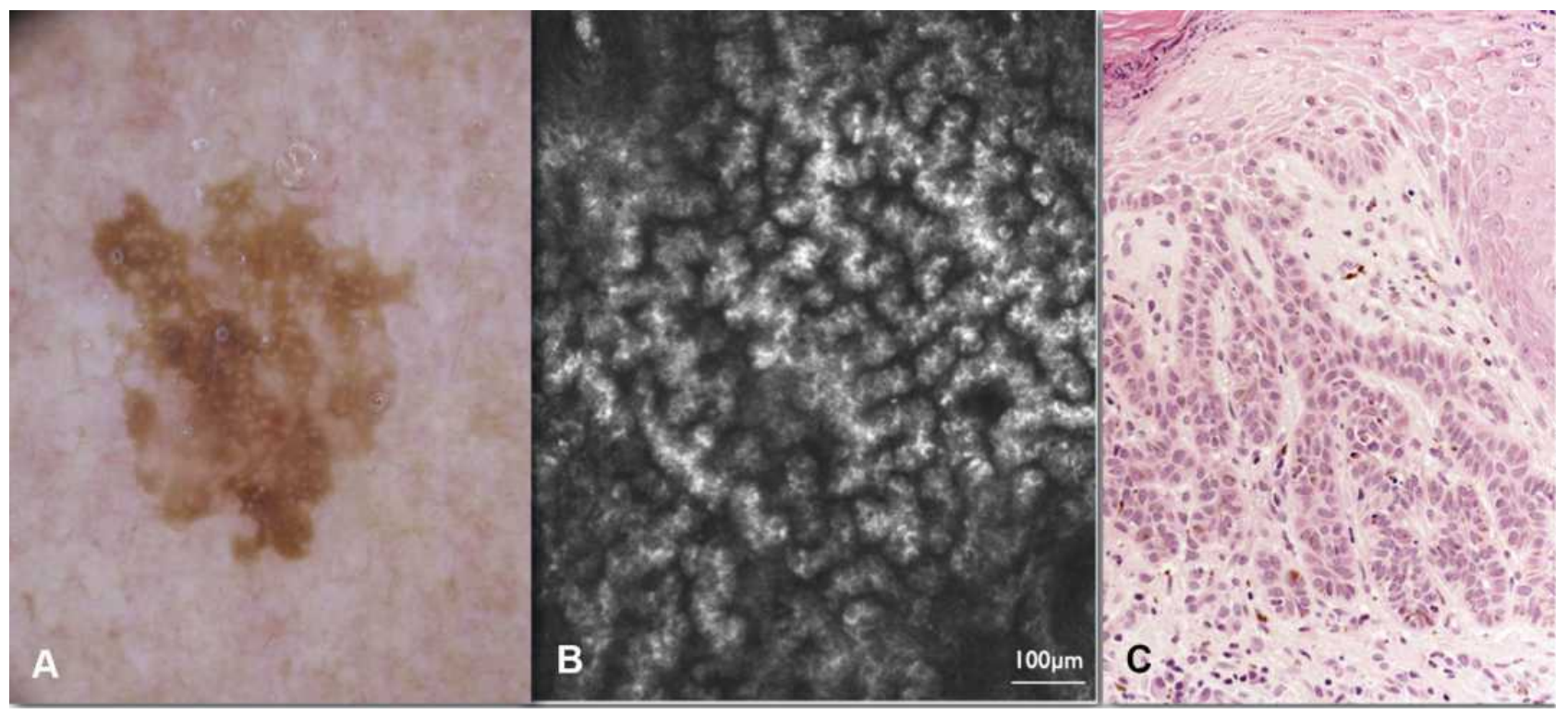

Figure 4 Adenoid or reticulated SK. (A) Dermoscopy of a lesion located on sun-damaged facial skin. A pseudo network is visible with well-demarcated borders. (B) In RCM, numerous bulbous projections at the level of DEJ and the so-called "cord like appearance" are seen. (C) Histology showing a basaloid epidermal proliferation arranged in thin, branched and intersected epithelial tracts (hematoxylin and eosin stain, original magnification 200x) 


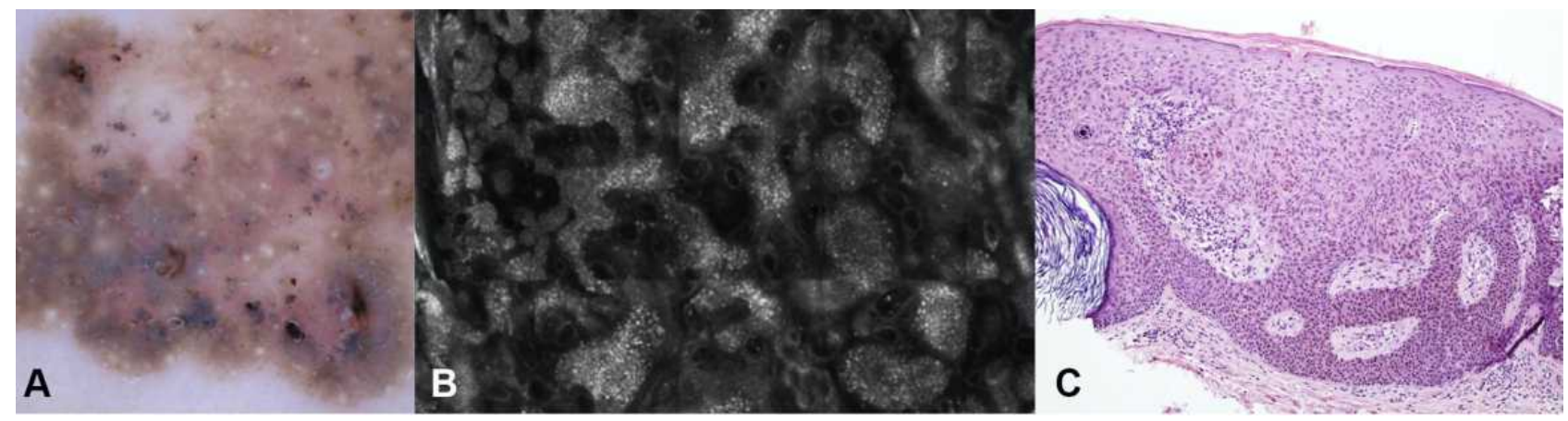

Figure 5 Clonal SK. (A) Dermoscopy with multiple grey blue globule like structures. This variant is difficult to differentiate from a pigmented basal cell carcinoma (B) RCM showing nests of epithelial cells. (C) Histology showing a basaloid epidermal proliferation characterized by acanthosis and horn pseudocyst. Melanin pigment is present in some cells. An intraepithelial roundish nest of epithelial cells is visible (Borst-jadassohn phenomenon) (hematoxylin and eosin stain, original magnification I00x).

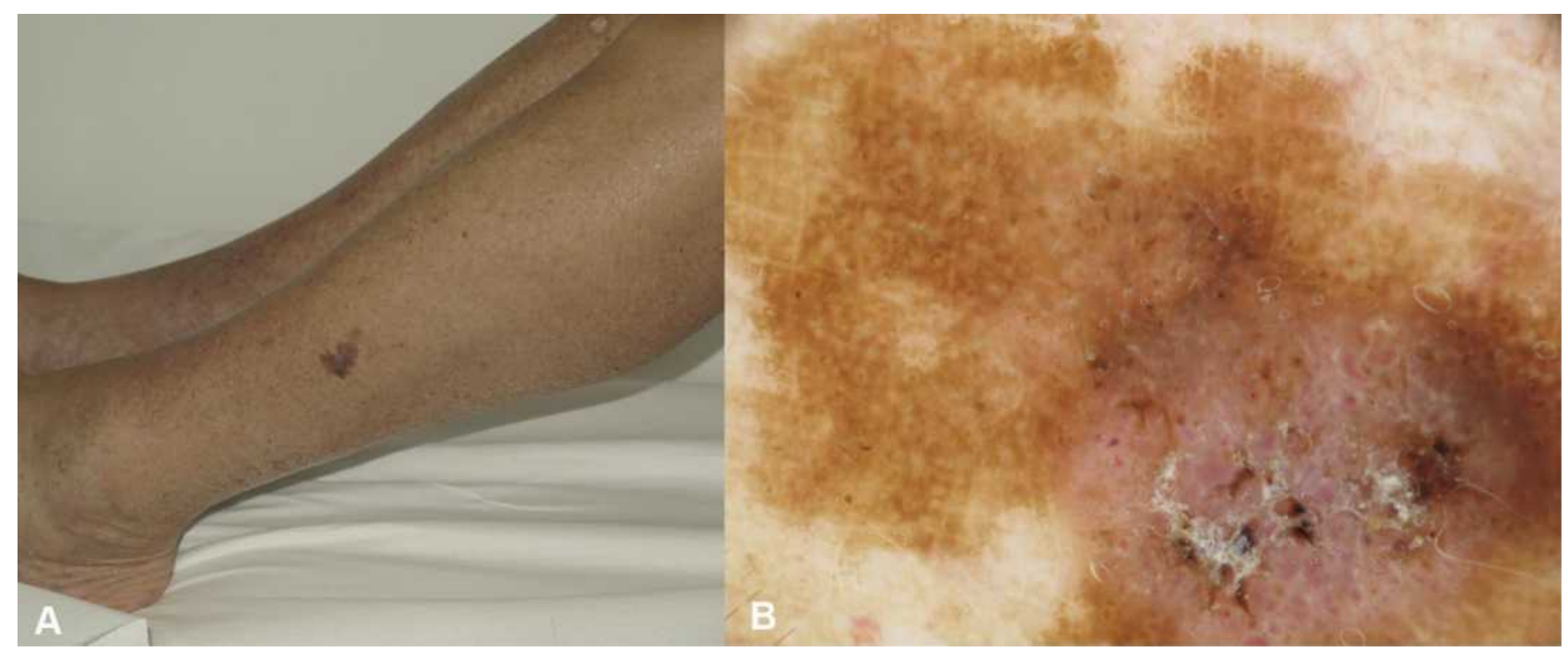

Figure 6 Irritated SK on the leg of a 76-year-old man. (A) Clinically the lesion is a large plaque with a partially keratotic surface, well-demarcated borders and color variegation from light brown to dark brown. (B) In dermoscopy a brown network is seen, with well-demarcated borders. In the non-pigmented area multiple dotted and hairpin vessels are visible surrounded by a whitish halo.

blue-gray globules, and leaf-like areas. In these cases, RCM and histology may be essential to the differential diagnosis.

\section{Challenging Variants of SK}

Clonal SK is the most subtle type where even dermoscopy may be not very conclusive. When pigmented, the intraepidermal nests appear as globule-like structures and are almost indistinguishable from the blue-gray globules of BCC and the dots/globules of melanocytic lesions. (Figure 5) In the non-pigmented variety, the presence of polymorphous vessels that are glomerular, linear-irregular and dotted along with a "white network" have been reported. The presence of comedo-like openings and milia- like cysts together with sharp lesion demarcation were reportedly helpful to diagnose clonal type SK; however, doubtful cases should be examined by RCM or histology. ${ }^{29}$

Irritated SK is characterized by hyperkeratosis with superficial scale crusts and keratinocytes may show foci of squamatization and squamous eddies. (Figure 6) There is evidence of increased apoptosis and lichenoid degeneration of the basal layer. ${ }^{1}$ Clinically, this results in the presence of scale, crust, and erosions, making the differential diagnosis from squamous cell carcinoma (SCC) challenging. A recent retrospective study investigated the dermoscopic criteria that could serve as potent predictors for the differential diagnosis between 


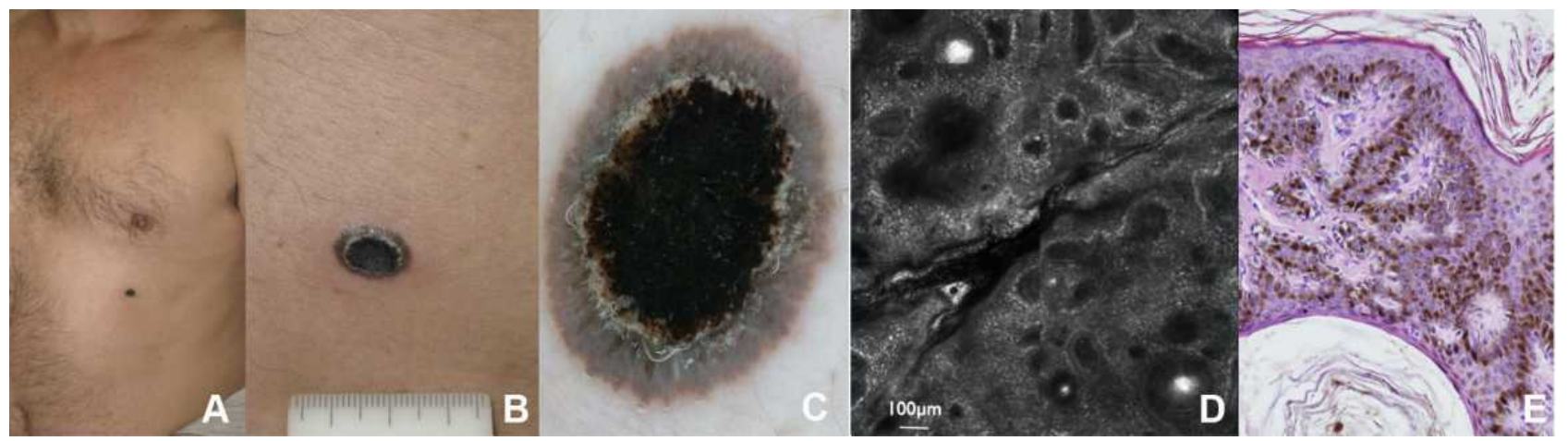

Figure 7 Melanoacanthoma. (A) A solitary dark papule on the abdomen of a 68-year-old man. (B) Close-up clinical image that shows the hyperkeratotic surface of the lesion. (C) In dermoscopy the lesion appears heavily pigmented, with peripheral streaks and a central hyperkeratosis. (D) RCM image at the level of the DEJ showing enlarged inter papillary spaces and multiple pseudo horn cysts. (E) Histology showing an SK with diffuse melanin pigmentation and colonization by melanocytes (hematoxylin and eosin, original magnification 200x).

irritated SK and SCC: predictors of SCC include dotted vessels, branched linear vessels, white structureless areas, white circles surrounding follicles, a diffuse irregular or peripheral vessel arrangement, and a central scale arrangement. In contrast, hairpin vessels, a diffuse regular vessel arrangement and multiple white halos surrounding vessels predict the diagnosis of irritated SK. ${ }^{30}$

Regressing SK may show a lichenoid pattern similar to that of lichen planus like keratosis (LPLK). Actually, it has been proposed that lichenoid keratosis represents an immunological or regressive response to pre-existing epidermal lesions, usually solar lentigines or seborrheic keratoses. Thus, regressing SK may represent the intermediate stage of this regressive phenomenon. ${ }^{31}$ Histologically, it is characterized by prominent band-like chronic inflammatory cells infiltrate in the upper dermis, pigment incontinence and individual degenerated keratinocytes in the epidermis. Dermoscopically, it exhibits a granular pattern typified by regularly distributed coarse blue grayish or brownish gray dots. When located on the face, it may be hard to differentiate this appearance from lentigo maligna.

Even pigmented acanthotic SK may be of difficult diagnosis when exhibit a starburst pattern, typical of Spitz nevus or spitzoid melanoma (Figure 7); it shows an intense dark blue pigmentation and the presence of pigmented streaks in a radial arrangement at the edge of the lesion, histopathological correlated to protrusions of the epidermis at the periphery of the lesion. $^{29}$
A retrospective study evaluated the dermoscopic features of a series of difficult-to-diagnose SKs that were biopsied for histopathological examination. Above the already cited atypical presentation of SK, more unusual dermoscopic patterns have been described. Bowenoid pattern is characterized by vascular structures like glomerular vessels, dotted vessels or a combination of both, while keratoacanthoma-like pattern is characterized by keratotic structures, blood crusts, pink color and linear/hairpin vascular structures. ${ }^{32}$ They are both difficult to differentiate from squamous cell carcinoma and histologic examination is often needed.

\section{Reflectance Confocal Microscopy}

Reflectance confocal microscopy (RCM) is a noninvasive imaging technique that allows to visualize the skin in vivo at a level of resolution similar to conventional histology. The limit in depth penetration is 250-300 micron, the image acquisition is in a horizontal plan of view and in a scale of grey color. ${ }^{33}$ Under RCM, SK has been described with its correlates with histology and dermoscopy. From a histopathologic point of view, the acanthotic pattern is the most frequent, in which a thick layer of basal cells is observed interspersed with pseudo-horny cysts. Invaginations to form keratin-filled pseudocysts are present. In RCM, the lesion surface presents epidermal projections, corresponding to the cerebriform appearance seen in dermoscopy (Figures 1 and figure 2). Keratin-filled surface invaginations or corneal plugs seen in RCM correspond to comedo-like openings in 
dermoscopy. Corneal pseudocysts can be found at various epidermal layers observed as white round structures that are highly refractile, they correspond to milia-like cysts in dermoscopy. The spinous-granular layer shows a regular appearance, either with a honeycombed or cobblestone pattern, depending on the degree of pigmentation. When acanthosis is present, a broadened honeycomb pattern can be seen, the keratinocytes appear regularly arranged, with thicker and brighter outlines (Figure 3). At the level of the dermal epidermal junction (DEJ), a ringed pattern can be usually observed, often with enlarged inter papillary spaces. Moreover, elongated bright tubular structures with bulbous projections can be seen at this level, they correlate with fingerprinting in dermoscopy. The vascular pattern of SK is made by looped and dilated vessels. ${ }^{33}$

Hyperkeratotic SK is only partially explorable in RCM. Because of the high degree of keratin on the lesion surface, the dermal epidermal junction is barely visible.

The reticulated or adenoid type of SK contains numerous thin tracts of basaloid epidermal cells that are branched and interwoven. They appear with numerous bulbous projections at the level of DEJ and the so-called "cord appearance" in which cords of epithelial cells are seen (Figure 4). They have less epidermal thickening, and horn pseudocysts usually are less prominent in reticulated seborrheic keratoses. Marked hyperpigmentation is often present, in RCM the keratinocytes appear intensely bright and usually with a cobblestone pattern of the spinous-granular layer.

The melanoacanthoma type is a histologic variant characterized by a high degree of pigmentation, in which an acanthotic variant of SK is associated with the proliferation of large dendritic melanocytes (Figure 7). In RCM, the melanoacanthoma type has been described in one anecdotical case, in which the presence of numerous bright dendritic cells in the epidermis and the hyperkeratotic surface did not allow to support the diagnosis of SK. ${ }^{34}$ Here in Figure 5 we show an example of melanoacanthoma, in which under RCM examination we were able to visualize a cobblestone pattern of the epidermis and to recognize the epithelial origin of the lesion thanks to the features seen at the level of the DEJ.

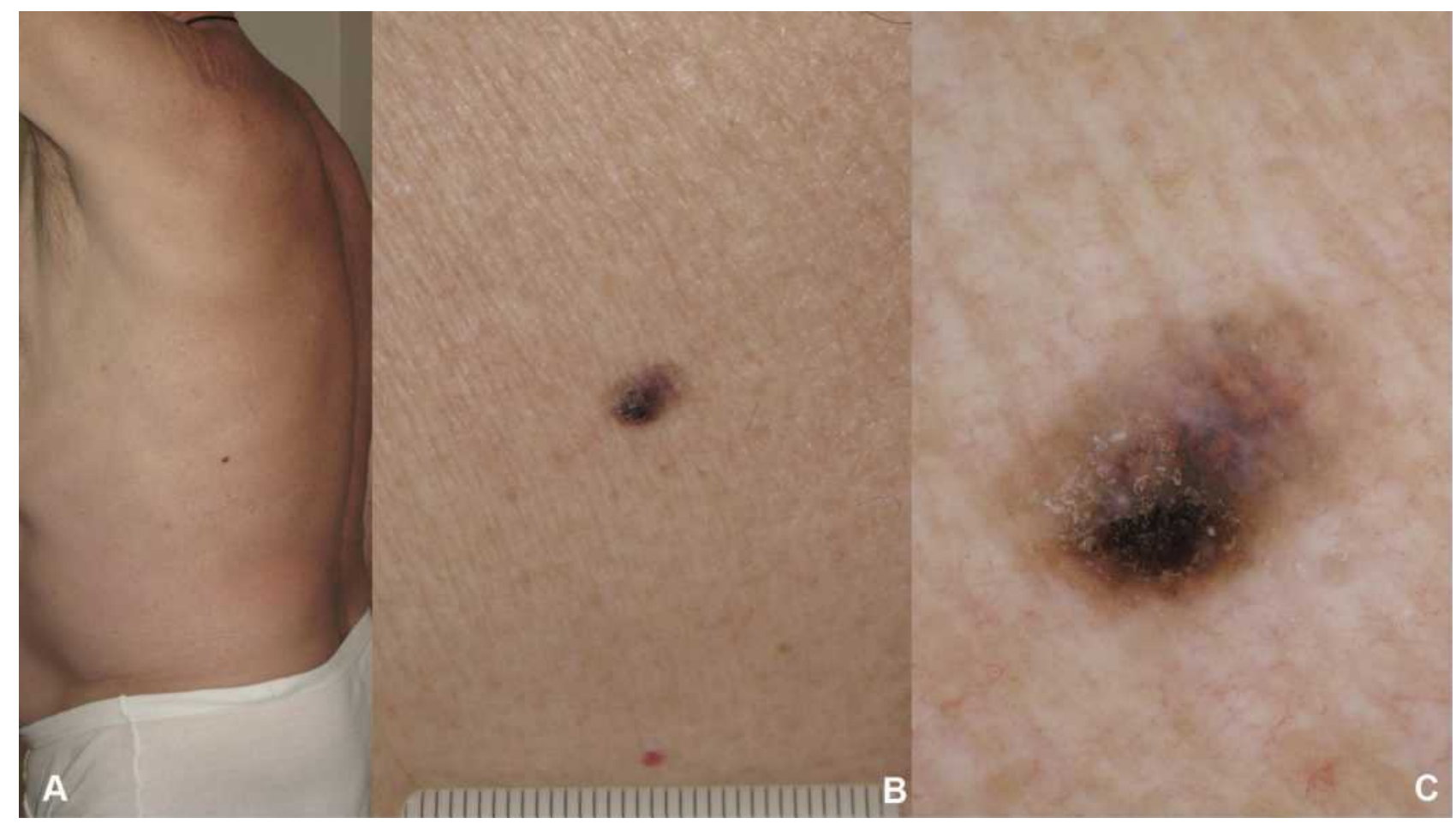

Figure 8 Melanoma mimicking an SK with regression. (A) Clinical view on the trunk of a 77-year-old man. (B) Close up showing a small dark brown, partially keratotic macule. (C) In dermoscopy the lesion is asymmetric, with a peripheral area with hyperkeratosis and the rest of the lesion showing grey color and peppering. The lesion was excised, histology revealed a in situ melanoma with regression. 
Clonal SK has been described in RCM that allows to visualize intraepidermal nesting of pigmented keratinocytes that permits to have a reliable in vivo diagnosis (Figure 5).

In LPLK, numerous plump bright cells are seen at the level of the superficial dermis together with remnants of the cordlike pattern typical on SK. However, when a reliable diagnosis of regressing SK cannot be made with confidence, a biopsy is needed in order to avoid misdiagnosis of a completely regressed melanoma (Figure 8).

When located on the face, flat SKs may require a careful differential diagnosis from lentigo maligna (LM). RCM is particularly useful, with the face region representing one of its best indications. A regular epidermal pattern, the absence of perifollicular infiltration of pagetoid melanocytes, the absence of DEJ nesting together with features typical of SK will support the diagnosis.

When traumatized, irritated SK may be difficult to diagnose clinically and dermoscopically.

Limits of RCM diagnosis of SKs have been highlighted in a study on 390 cases. In this study, over 390 patients were included with a clinical diagnosis of SK, of these, $258(66.2 \%)$ patients were diagnosed with SK, 97 of 390 (24.9\%) patients could not be diagnosed by the dermatologist according to RCM. Of all 37 biopsied lesions, 23 were SK, 6 were actinic keratosis, 2 were basal cell carcinoma, and 2 were squamous cell carcinoma. The authors conclude that this could be due to the limit in depth penetration of $\mathrm{RCM}^{35}$ (Table 1).

\section{Management}

SK can be managed conservatively when the diagnosis is clear cut and they do not represent a reason of concern for the patient. The sudden appearance of multiple pruritic SKs is known as the Leser-Trélat sign. ${ }^{36}$ This is a paraneoplastic condition that has been associated with the development of adenocarcinoma of the gastrointestinal tract, lymphoma, Sézary syndrome, and acute leukemia. A pseudo Leser-Trélat sign has also been reported, defined as the sudden onset of pruritus and erythema involving pre-existing multiple SKs during chemoterapy. ${ }^{37}$

The treatment in practice is mainly minor surgery, including cryosurgery, shave excisions, and laser-assisted removal.

Cryotherapy is the easier to perform and fast treatment, that can be used to safely remove multiple SKs at a same time. In a survey among 594 board certified dermatologists from the US, participants declared to treat SKs with cryosurgery as a preferred method. ${ }^{38}$ Patient's satisfaction has been evaluated in a small study in which curettage was also employed, with patients preferring cryotherapy over this other method. ${ }^{39}$

Other treatment options include shave excision, electrodessication, or a combination of these.

Several laser treatments can be employed. A comparative trial of 42 patients with SKs located on the back, chest, face, and neck compared Er:YAG (erbiumdoped yttrium aluminium garnet) laser and cryotherapy head to head on the same patient. In the same session, half of the lesions were treated with cryotherapy and the other half were treated with Er:YAG laser. Following the first treatment, complete healing was detected in all of the lesions $(100 \%)$ treated with Er:YAG lasers, the healing rate was $68 \%$ in the cryotherapy group $(P<0.01)$. In the

Table I List of Dermoscopic and RCM Features of the Main SK Subtype. Of Note, Milia-Like Cysts and Comedo-Like Openings Can Be Found in All Subtypes

\begin{tabular}{|l|l|l|}
\hline SK Subtype & Dermoscopy & RCM \\
\hline Acanthotic & Milia like cysts and comedo-like openings, cerebriform surface & Acanthosis, Pseudo-horn cysts, fissures and ridges \\
\hline Hyperkeratotic & Crusts and scales and cerebriform surface & Crusts, scales, fissures and ridges \\
\hline Reticulated & Sharply demarcated borders and brown network & Cords and bulbous projections \\
\hline Irritated & Scales, crusts, erosions, hairpin vessels & Scales, hyperkeratosis, inflammatory cells, looped vessels \\
\hline Lichenoid & Peppering & $\begin{array}{l}\text { Remnants of cords and multiple melanophages in the } \\
\text { dermis }\end{array}$ \\
\hline Clonal & Blue-grey nests & Nests of epithelial cells \\
\hline Melanoacanthoma & $\begin{array}{l}\text { Intense black pigmentation, at times pigmented streaks at the } \\
\text { periphery of the lesion }\end{array}$ & $\begin{array}{l}\text { Bright dendritic cells in the surface and melanophages } \\
\text { in the dermis }\end{array}$ \\
\hline
\end{tabular}


Er:YAG laser-treated group, hyperpigmentation was significantly lower than in the cryotherapy group. ${ }^{40}$

The $\mathrm{CO}_{2}$ laser is an alternative option but with a slightly higher risk of scarring and pigmentary changes. Small case series have reported the use of intense pulsed light, pulsed-dye laser, Nd:YAG (neodymium-doped yttrium aluminum garnet) laser, or the 755-nm alexandrite picosecond laser. ${ }^{41-44}$ None of these studies was specifically designed for elderly patients, head-to-head comparison of the various laser treatments are missing.

Among medical treatments, hydrogen peroxide $40 \%$ (HP40) was approved by the US Food and Drug

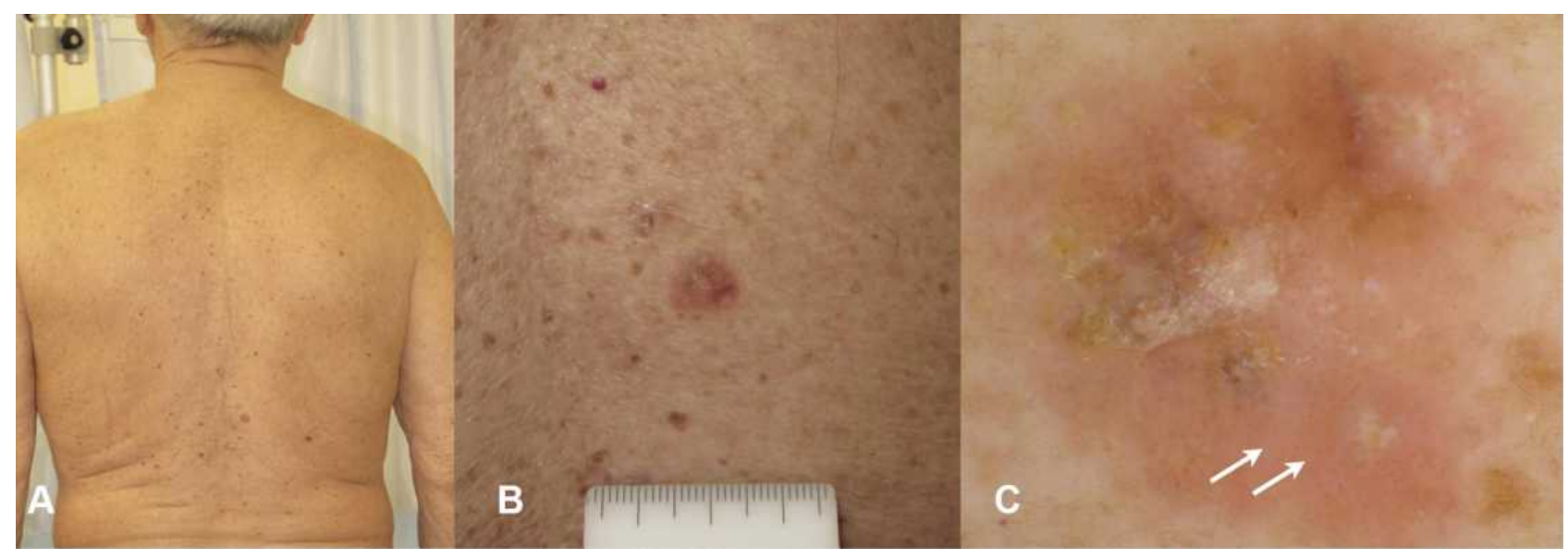

Figure 9 Melanoma mimicking an irritated SK. (A) Clinical view of the back of a 78-year-old man with multiple SKs, cherry angiomas and nevi. (B) Clinical close-up image, the lesion appears erythematous and not well defined. (C) In dermoscopy dotted vessels are visible over the entire lesion area (white arrows). Scales and crust are present on the surface.
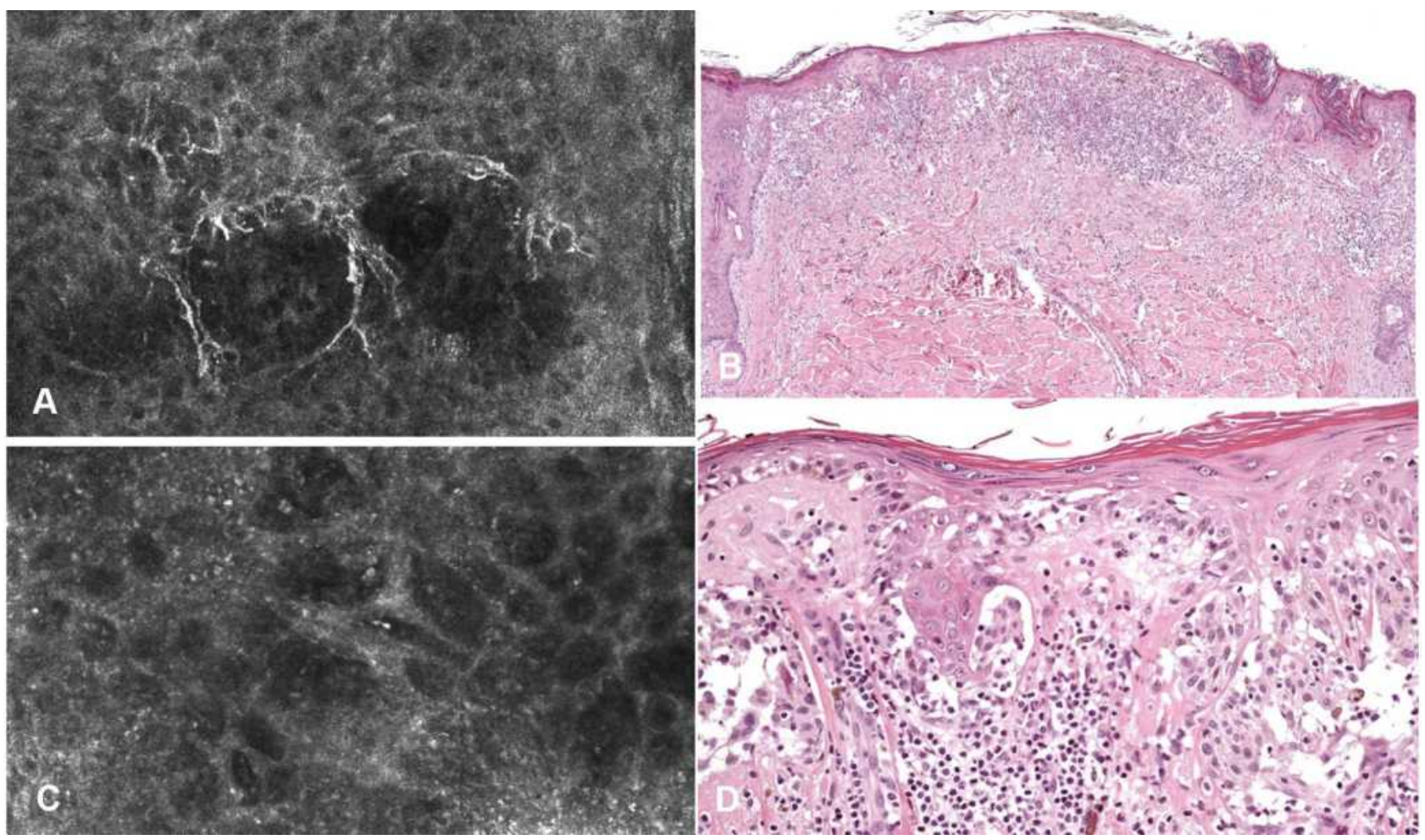

Figure 10 RCM and histologic imaging of the case in Figure 9. (A) RCM at the level of the spinous layer showing bright dendritic cells. (B) Histology showing an irregular and asymmetrical junctional proliferation of melanocytes, characterized by consumption of thinned epidermis and a dense dermal lymphocytic infiltration. Some epidermal invaginations filled with keratin are evident on the right (hematoxylin and eosin stain, original magnification 40x). (C) RCM mosaic at the level of the DEJ showing non edged papillae and meshwork pattern. (D) Histology showing atypical melanocytes arranged in a lentiginous pattern and confluent irregular nests (hematoxylin and eosin stain, original magnification 200x)(Figure 10, Figure II). 
Administration for topical treatment of SK in December 2017. HP40 may act through its direct oxidation of organic tissues, generation of reactive oxygen species, and local lipid peroxidation and also by the generation of local concentrations of oxygen that are toxic to SK cells and induce apoptosis. In a trial of 937 patients with 4 SKs each, patients were randomized 1:1 to HP40 or vehicle. ${ }^{45}$ The treatment efficacy was highest on facial lesions (clear or near-clear in 65\%) followed by truncal SKs (46\%) and SKs on the extremities (38\%). However, the optimal number of treatment sessions was not evaluated. ${ }^{45}$

\section{Conclusions}

Even if SKs are very common in the elderly and constitute a common reason for consultation in the daily practice, they may be at times challenging. False positive and false negative cases are not exceptional. The worrisome scenario of misdiagnosing a nodular melanoma could be avoided by applying dermoscopy. As highlighted by Carrera et $\mathrm{al}^{23}$ the majority of melanomas (MM) clinically resembling SKs could have been correctly diagnosed applying dermoscopy. Despite the verrucous appearance, most of the melanomas in the series by Carrera et $\mathrm{al}^{23}$ showed at least one melanomaspecific criterion, the more frequent being blue-black sign, pigment network, pseudopods or streaks, and/or blue-white veil (Figures 9-11). Melanoma should be excluded also when evaluating SKs located on the face. SKs frequently arise on the head/neck area, where the reticular or adenoid type of SK is more common. On the other hand, SKs may mimic malignant tumors, especially those hyper pigmented, reticular and irritated (Figure 12). Dermoscopy and RCM have been demonstrated to improve diagnostic accuracy and help recognizing false-positive cases. The main limitation of dermoscopy is the evaluation of regressing lesions, in RCM one limit could be a high degree of hyperkeratosis of the lesions hampering the visualization of the DEJ.

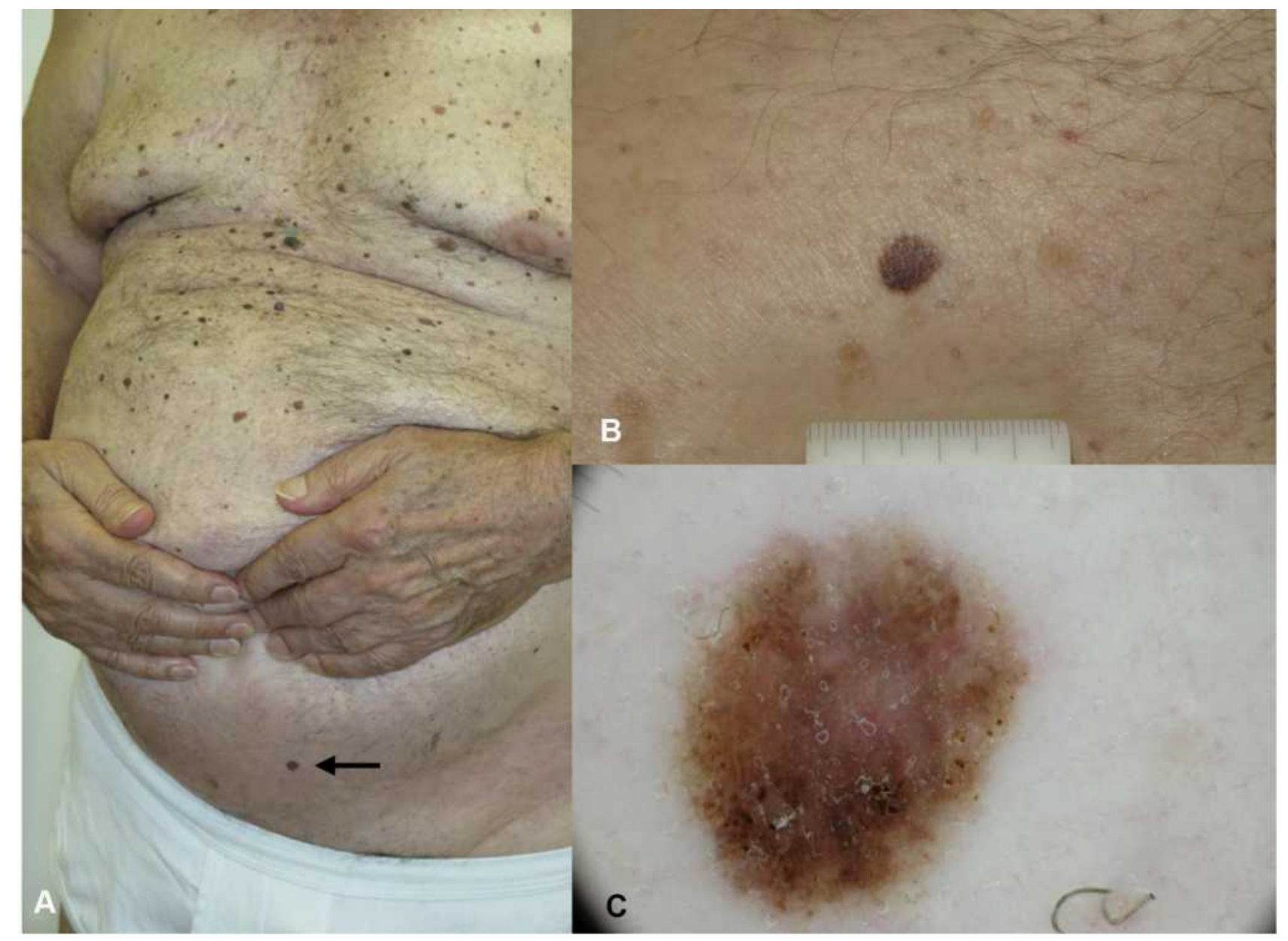

Figure I I Invasive melanoma on the abdomen of an $8 \mathrm{I}$-year-old man. (A) The patient had multiple nevi and multiple SKs. All lesions were examined with dermoscopy, also the "hidden" ones. (B) Close up of the $7 \mathrm{~mm}$ macule finally diagnosed as melanoma. (C) In dermoscopy asymmetry and irregular globules are seen. Histologically a diagnosis of $0.6 \mathrm{~mm}$ Breslow thickness melanoma was rendered. 


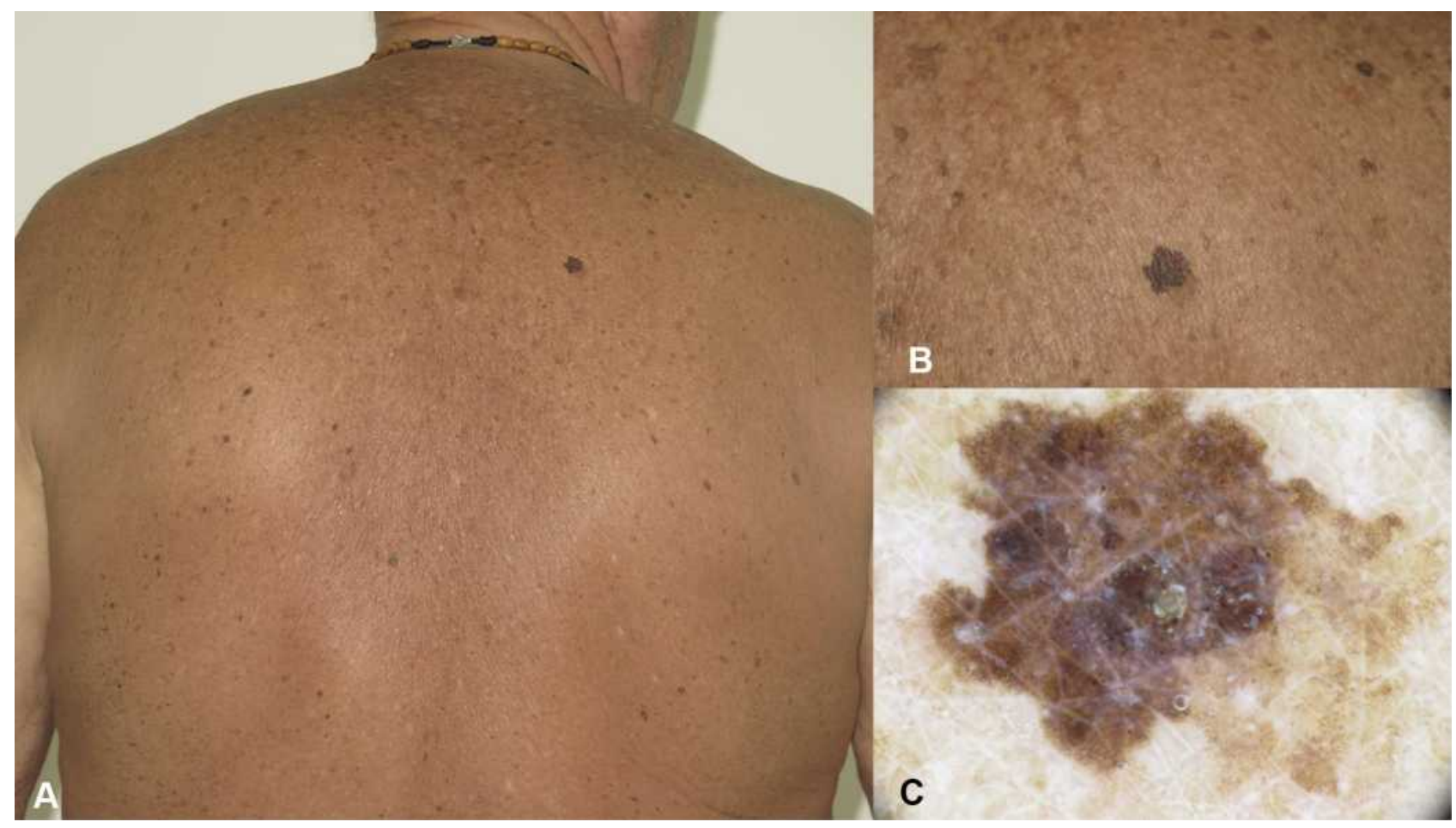

Figure 12 SK mimicking melanoma. (A) Multiple solar lentigos and SKs on the back of a 69-year-old man. (B) One larger, outstanding macule with color variegation. (C) In dermoscopy, the lesion is characterized by a very regular network, with well demarcated and moth-eaten borders. Hyperkeratosis can be seen on the lesion surface.

A conservative approach is usually preferred in elderly patients, in case of cosmetic and/or functional discomfort several treatment options are available, including cryotherapy and minor surgery.

\section{Disclosure}

The authors report no conflicts of interest in this work.

\section{References}

1. Brenn T, Elgart GW, Howard V, et al. Benign acanthomas/keratoses. In: Elder DE, Massi D, Scolyer RA, Willemze R, editors. WHO Classification of Skin Tumours. 4th. Lyon: IARC; 2018:57-63.

2. Memon AA, Tomenson JA, Bothwell J, Friedmann PS. Prevalence of solar damage and actinic keratosis in a Merseyside population. $\mathrm{Br}$ $J$ Dermatol. 2000;142(6):1154-1159. doi:10.1046/j.1365-2133.2000. 03541.x

3. Yeatman JM, Kilkenny M, Marks R. The prevalence of seborrhoeic keratoses in an Australian population: does exposure to sunlight play a part in their frequency? $B r J$ Dermatol. 1997;137(3):411-414. doi:10.1111/j.1365-2133.1997.tb03748.x

4. Braun RP, Rabinovitz HS, Krischer J, et al. Dermoscopy of pigmented seborrheic keratosis: a morphological study. Arch Dermatol. 2002;138 (12):1556-1560. doi:10.1001/archderm.138.12.1556

5. Papageorgiou V, Apalla Z, Sotiriou E, et al. The limitations of dermoscopy: false-positive and false-negative tumours. J Eur Acad Dermatol Venereol. 2018;32(6):879-888. doi:10.1111/jdv.14782

6. Argenziano G, Rossiello L, Scalvenzi M, et al. Melanoma simulating seborrheic keratosis: a major dermoscopy pitfall. Arch Dermatol. 2003;139:389-391. doi:10.1001/archderm.139.3.389-b
7. Braga JC, Scope A, Klaz I, et al. Melanoma mimicking seborrheic keratosis: an error of perception precluding correct dermoscopic diagnosis. J Am Acad Dermatol. 2008;58(5):875-880. doi:10.1016/ j.jaad.2007.12.011

8. Carrera C, Segura S, Palou J, et al. Seborrheic keratosis-like melanoma with folliculotropism. Arch Dermatol. 2007;143:373-376. doi:10.1001/archderm.143.3.373

9. Ahlgrimm-Siess V, Cao T, Oliviero M, et al. Seborrheic keratosis: reflectance confocal microscopy features and correlation with dermoscopy. J Am Acad Dermatol. 2013;69(1):120-126. doi:10. 1016/j.jaad.2012.12.969

10. Tindall JP, Smith JG Jr. Skin lesions of the aged and their association with internal changes. JAMA. 1963;186(12):1039-1042. doi:10.1001/jama.1963.03710120021004

11. Hafner C, Landthaler M, Mentzel T, Vogt T. FGFR3 and PIK3CA mutations in stucco keratosis and dermatosis papulosa nigra. $\mathrm{Br}$ J Dermatol. 2010;162(3):508-512. doi:10.1111/j.1365-2133.2009.09 488.x

12. Lin J, Han S, Cui L, et al. Evaluation of dermoscopic algorithm for seborrhoeic keratosis: a prospective study in 412 patients. J Eur Acad Dermatol Venereol. 2014;28(7):957-962. doi:10.1111/ jdv. 12241

13. Kopf AW, Rabinovitz H, Marghoob A, et al. "Fat fingers:" a clue in the dermoscopic diagnosis of seborrheic keratoses. $\mathrm{J}$ Am Acad Dermatol. 2006;55(6):1089-1091. doi:10.1016/j.jaad.2006.08.028

14. Steiner A, Konrad K, Pehamberger H, et al. Verrucous malignant melanoma. Arch Dermatol. 1988;124:1534-1537. doi:10.1001/ archderm.1988.01670100036009

15. Zabel RJ, Vinson RP, McCollough ML. Malignant melanoma arising in a seborrheic keratosis. J Am Acad Dermatol. 2000;42(5):831-833. doi:10.1067/mjd.2000.103634

16. Yakar JB, Sagi A, Mahler D, et al. Malignant melanoma appearing in seborrheic keratosis. J Dermatol Surg Oncol. 1984;10(5):382-383. doi:10.1111/j.1524-4725.1984.tb00481.x 
17. Ohnishi T, Hamano M, Watanabe S. Clinically verrucous and histologically discohesive melanoma. A case report with dermoscopic and immunohistochemical observations. Australas J Dermatol. 2014;55 (2):e21-e23. doi:10.1111/ajd.12023

18. Salerni G, Alonso C, Gorosito M, et al. Seborrheic keratosis-like melanoma. J Am Acad Dermatol. 2015;72(1):S53-S55. doi:10.1016/ j.jaad.2014.07.009

19. Tran PT, Truong AK, Munday W, et al. Verrucous melanoma masquerading as a seborrheic keratosis. Dermatol Online J. 2019;25(2). doi:13030/qt1m07k7fm

20. Brandão ML, Oliveira Lima CM, Moura HH, et al. Dermatoscopic findings of seborrheic keratosis in Melanoma. Acta Dermatovenerol Croat. 2016;24(2):144-147.

21. Longo C, Moscarella E, Piana S, et al. Not all lesions with a verrucous surface are seborrheic keratoses. J Am Acad Dermatol. 2014;70(6):e121-e123. doi:10.1016/j.jaad.2013.10.042

22. Izikson L, Sober AJ, Mihm MC Jr, et al. Prevalence of melanoma clinically resembling seborrheic keratosis: analysis of 9204 cases. Arch Dermatol. 2002;138:1562-1566. doi:10.1001/archderm.138.12.1562

23. Carrera C, Segura S, Aguilera P, et al. Dermoscopic clues for diagnosing melanomas that resemble seborrheic keratosis. JAMA Dermatol. 2017;153 (6):544-551. doi:10.1001/jamadermatol.2017.0129

24. Kurihara Y, Furue M. Occult basal cell carcinoma arising in seborrheic keratosis. Case Rep Dermatol. 2019;11(1):48-51. doi:10.1159/ 000497067

25. Endoh K, Ohara M, Kosegawa G, et al. Occult basal cell carcinoma arising in seborrheic keratosis. J Dermatol. 1998;25(6):374-378. doi:10.1111/j.1346-8138.1998.tb02417.x

26. Gao -Y-Y-Y-Y, An X-J, Yang J, et al. Seborrheic keratosis mimicking basal cell carcinoma under dermoscopy: a case report. Chin Med J. 2020;133(17):2139-2140. doi:10.1097/CM9.0000000000001010

27. Yanagihara S, Yoshida Y, Tsuruta D, et al. Basal cell carcinoma showing surface hyperkeratosis clinically mimicking seborrheic keratosis. J Dermatol. 2015;42(12):1195-1196. doi:10.1111/1346-8138.13100

28. Lombardi M, Pampena R, Borsari S, et al. Dermoscopic features of basal cell carcinoma on the lower limbs: a chameleon! Dermatology. 2017;233(6):482-488. doi:10.1159/000487300

29. Minagawa A, Tanaka M, Koga H, et al. Pigmented seborrheic keratosis showing starburst pattern. J Am Acad Dermatol. 2016;75(1): e11-e13. doi:10.1016/j.jaad.2015.12.048

30. Papageorgiou C, Spyridis I, Manoli SM, et al. Accuracy of dermoscopic criteria for the differential diagnosis between irritated seborrheic keratosis and squamous cell carcinoma. J Am Acad Dermatol. 2020:S0190-9622(20)30227-9.

31. Zaballos P, Blazquez S, Puig S, et al. Dermoscopic pattern of intermediate stage in seborrhoeic keratosis regressing to lichenoid keratosis: report of 24 cases. Br J Dermatol. 2007;157(2):266-272. doi:10.1111/j.1365-2133.2007.07963.x

32. Squillace L, Cappello $\mathrm{M}$, Longo $\mathrm{C}$, et al. Unusual dermoscopic patterns of seborrheic keratosis. Dermatology. 2016;232(2):19 8-202. doi:10.1159/000442439
33. Shahriari N, Grant-Kels JM, Rabinovitz H, Oliviero M, Scope A. Reflectance confocal microscopy: diagnostic criteria of common benign and malignant neoplasms, dermoscopic and histopathologic correlates of key confocal criteria, and diagnostic algorithms. J Am Acad Dermatol. 2021;84(1):17-31. doi:10.1016/j.jaad.2020.05.154

34. Porto ACS, Blumetti TP, Macedo MP, Braga JCT. Melanoacanthoma: a potential pitfall of reflectance confocal microscopy. An Bras Dermatol. 2019;94(6):747-750. doi:10.1016/j.abd.2019.01.002

35. Guo A, Chen J, Yang C, Ding Y, Zeng Q, Tan L. The challenge of diagnosing seborrheic keratosis by reflectance confocal microscopy. Skin Res Technol. 2018;24(4):663-666. doi:10.1111/srt.12582

36. Sperry K, Wall J. Adenocarcinoma of the stomach with eruptive seborrheic keratoses: the sign of Leser-Trélat. Cancer. 1980;45 (9):2434-2437. doi:10.1002/1097-0142(19800501)45:9<2434::aidcncr2820450932>3.0.co;2-m

37. Husain Z, Ho JK, Hantash BM. Sign and pseudo-sign of Leser-Trélat: case reports and a review of the literature. J Drugs Dermatol. 2013;12(5):e79-e87.

38. Jackson JM, Alexis A, Berman B, Berson DS, Taylor S, Weiss JS. Current understanding of seborrheic keratosis: prevalence, etiology, clinical presentation, diagnosis, and management. J Drugs Dermatol. 2015;14(10):1119-1125.

39. Wood LD, Stucki JK, Hollenbeak CS, Miller JJ. Effectiveness of cryosurgery vs curettage in the treatment of seborrheic keratoses. JAMA Dermatol. 2013;149(1):108-109. doi:10.1001/ 2013.jamadermatol.275

40. Gurel MS, Aral BB. Effectiveness of erbium: YAGlaser and cryosurgery in seborrheic keratoses: randomized, prospective intraindividual comparison study. J Dermatolog Treat. 2015;26(5):477-480. doi:10. 3109/09546634.2015.1024597

41. Wollina U. Erbium-YAG laser therapy - analysis of more than 1200 treatments. Glob Dermatol. 2016;3(2):268-272. doi:10.15761/god.10 00171

42. Sayan A, Sindel A, Ethunandan M, et al. Management of seborrhoeic keratosis and actinic keratosis with an erbium: YAGlaser-experience with 547 patients. Int J Oral Maxillofac Surg. 2019;48(7):902-907. doi:10.1016/j.ijom.2018.08.008

43. Bruscino N, Conti R, Campolmi P, et al. Dermatosis papulosa nigra and 10,600-nm CO 2 laser, a good choice. J Cosmet Laser Ther. 2014;16(3):114-116. doi:10.3109/14764172.2013.854640

44. Cevizci R, Bezgin SÜ, Altın G, et al. Treatment of seborrheic keratosis in bilateral external auditory canal using fiber $\mathrm{CO} 2$ laser. Kulak Burun Bogaz Ihtis Derg. 2016;26(5):304-306. doi:10.5606/ kbbihtisas.2016.04810

45. Baumann LS, Blauvelt A, Draelos ZD, et al. Safety and efficacy of hydrogen peroxide topical solution, $40 \%(\mathrm{w} / \mathrm{w})$, in patients with seborrheic keratoses: results from 2 identical, randomized, double-blind, placebo-controlled, Phase 3 studies (A-101-SEBK -301/302). J Am Acad Dermatol. 2018;79(5):869-877. doi:10.1016/ j.jaad.2018.05.044
Clinical, Cosmetic and Investigational Dermatology is an international, peer-reviewed, open access, online journal that focuses on the latest clinical and experimental research in all aspects of skin disease and cosmetic interventions. This journal is indexed on CAS.
The manuscript management system is completely online and includes a very quick and fair peer-review system, which is all easy to use. Visit http://www.dovepress.com/testimonials.php to read real quotes from published authors. 